\title{
An industry update: what are the latest developments in the field of therapeutic delivery? Industry Update: 1st December to 31st December 2016
}

First draft submitted: 15 January 2017; Accepted for publication: 18 January 2017; Published online: 22 February 2017

Keywords: acquisitions and mergers $\bullet$ clinical trials $\bullet$ collaborations $\bullet$ pipeline news

December 2016, the period covered by this article, was characterized by a number of meetings where news on advances in company pipelines were disclosed, including those of the American Society of Hematology (ASH) meeting, the American Association for Cancer Research San Antonio Breast Cancer Symposium, the World Conference on Lung Cancer (WCLC), the Clinical Trials on Alzheimer's Disease meeting and the European Society for Medical Oncology Asia Congress. Information from these meetings provides for most of the last section of this article on updates of therapeutic advances from clinical trials. There were also some interesting merger and acquisition news items, in particular the back and forth interaction between Actelion and Johnson and Johnson regarding a possible strategic transaction between the two companies, which at the time of writing was still under discussion.

It was a good month for success with newer therapeutic delivery options becoming commercial reality, as exemplified by the regulatory approval of an antisense oligonucleotide product, nusinersen (Spinraza ${ }^{\circledR}$, Biogen and Ionis Pharmaceuticals) and the initiation of filing for regulatory approval for a CAR T-cell therapy product, axicabtagene ciloleucel (Kite Pharma, CA, USA).

Virtually all of material for this article was sourced from press releases issued by the various companies mentioned.

\section{Business news}

\section{Acquisitions, mergers}

\section{Actelion \& Johnson \& Johnson}

There was big Pharma involved in corporate courtship activity through mid to late December 2016, with a late November 2016 approach to Actelion (Allschwil, Switzerland) by Johnson \& Johnson (NJ, USA) regarding a potential strategic transaction [1] that went cold mid-month, with Johnson and Johnson indicating it had withdrawn from discussion and Actelion acknowledging that change $[2,3]$. Then there was a report that Sanofi (Paris, France) had engaged in talks to acquire Actelion [4] which neither company officially commented upon, but discussions with Johnson and Johnson opened up again toward the end of December, with Actelion entering into exclusive negotiations with the US giant $[5,6]$. As of the time of writing the potential transaction remains under discussion. Actelion may be of interest to potential acquirers as it has marketed products in a well-defined area of cardiovascular disease (pulmonary arterial hypertension, based on endothelin receptor antagonists) and in specialty areas, for example a product for topical treatment of mycosis fungoides-type cutaneous T-cell lymphoma (CTCL), and also a genetic disease therapy approved in different territories for type I Gaucher disease and Niemann-Pick Type C (NP-C) disease. The company has an interesting development

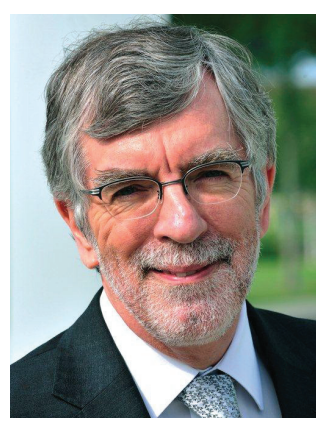

Peter Timmins Department of Pharmacy, School of Applied Sciences, University of Huddersfield, Huddersfield HD1 3DH, UK Author for correspondence: p.timmins@hud.ac.uk 
pipeline, including a selective S1P1 receptor modulation therapy targeting multiple sclerosis and lupus and in orexin receptor antagonism, targeted for therapy of certain CNS disorders.

\section{Novartis \& Ziarco}

Novartis (Basel, Switzerland) added an investigational oral histamine $\mathrm{H}_{4}$ receptor antagonist to its dermatology portfolio, targeting eczema (atopic dermatitis) through the acquisition of the Canterbury, UK-based Ziarco Group. There is currently no safe and effective, well-tolerated oral therapy for moderate to severe eczema, so this acquisition enables the further clinical progress of this compound as well as additional, less advanced assets. Ziarco had been formed in 2012 with assets originating from Pfizer, and the advanced candidate that promoted the interest from Novartis, ZPL389, has demonstrated clinically and statistically significant reductions in eczema at the 8-week treatment point in a proof of concept study in 98 patients [7].

\section{Novartis \& Encore Vision}

Presbyopia is a common vision defect, manifesting as the natural, gradual loss of ability to focus on near objects, and becomes noticeable usually in subjects over 40 years of age. It is associated with stiffening of the lens of the eye, thus impacting accommodation and hence difficulties in focusing. Encore Vision had completed Phase I-II studies of its lead product EV06, lipoic acid choline ester $1.5 \%$ ophthalmic solution, early in 2016 and demonstrated statistically significant differences to placebo in distant corrected near vision over the 90-day course treatment of the trials. Lipoic acid choline ester is a prodrug that is broken down in the eye and the metabolite dehydrolipoic acid breaks disulfide bonds between lens proteins to restore lens flexibility [8]. The acquisition of Encore Vision announced by Novartis [9] brings a first-in-class disease-modifying topical therapy to the Novartis ophthalmology pipeline, potentially enabling treatment of a high prevalence disease where there is high unmet need.

\section{Northern Biologics \& Mosaic Biomedicals}

The further development of a humanized antibody, MSC-1, that targets leukemia inhibitory factor, which is overexpressed in certain solid tumors and promotes cancer progression, will be enabled, and accelerated by the merger of Northern Biologics Inc. (Toronto, Canada) and Mosaic Biomedicals SL (Barcelona, Spain) [10]. The deal is supported by venture capital from Versant Ventures, which already has interests in both companies. Celegne Corp has exercised an option to acquire certain rights to MSC-1 under its existing arrangement with Northern Biologics, and the merged business now has access to full funding of the early clinical development of MSC-1 and also a preclinical portfolio of therapeutic antibodies. Clinical trials of MSC-1 are expected to commence during 2017.

\section{Collaborations}

\section{AstraZeneca \& Bicycle Therapeutics}

Bicycle Therapeutics (Cambridge, UK) have developed highly constrained bicyclic small (9-15 amino acids) peptides that have antibody-like target specificity and high affinity against diverse targets and target classes. They have large molecular surface area allowing them to modulate protein-protein interactions, but unlike antibodies can rapidly penetrate tissues, are likely not to show immunogenicity, are water soluble and so amenable to different formulation options and can be created with desirable pharmacokinetic properties that can help avoid certain toxicity risks [11]. The Cambridge company announced its collaboration with AstraZeneca to develop therapeutics based on its bicyclic peptide product platform, known as Bicycle ${ }^{\circledR}$, for the treatment of respiratory, cardiovascular and metabolic diseases. Under the terms of the collaboration, the peptides will be discovered by Bicycle Therapeutics and then AstraZeneca will be responsible for further development and product commercialization. Bicycle Therapeutics will receive upfront payments, future R\&D funding, milestone payments and royalties [11].

\section{Pfizer \& IBM}

Pfizer (NY, USA) and IBM Watson Health (NY, USA) announced their collaboration, where Pfizer will use IBM Watson for Drug Discovery to analyze large amounts of data from various sources - both from the public domain and of internal Pfizer origin - to help generate insight in the search for and the development of novel immune-oncology therapeutics. An IBM Watson spokesperson noted that medical innovation can arise as researchers find new patterns in existing knowledge, and so new tools such as Watson for Drug Discovery were needed to help navigate the large amounts of data that can now be accessed [12].

\section{Takeda \& Lightstone Ventures}

The creation of Cerevance, a neuroscience company aiming to discover and develop novel therapeutics for neurological and psychiatric disorders, was jointly announced by Takeda Pharmaceutical Company (Osaka, Japan) and Lightstone Ventures (MA, USA) [13]. Takeda is providing a 25-person neuroscience R\&D team from its former Cambridge, UK site, fully-equipped laboratory space and licenses to 
a portfolio of preclinical and clinical stage drug programs. Start-up funding for the new company is being provided by both Takeda and Lightstone. The establishment of Cerevance provides a home for some of the most promising scientists, and a continuation of the most interesting programs in neuroscience that Takeda had in the UK prior to it announcing the closure of its Cambridge site and represents an example of Takeda's $\mathrm{R} \& \mathrm{D}$ strategy of enabling and then leveraging external innovation.

\section{Sanofi \& JHL Biotech}

The importance of China as a growing strategic market and development base for innovative pharmaceuticals was underlined by the announcement by Sanofi and JHL Biotech (Shanghai, China) of their strategic biologics alliance to develop and commercialize biological therapeutics in China, with potential for international expansion [14]. Under the terms of the collaboration, Sanofi will acquire exclusive rights to a proposed rituximab biosimilar developed by JHL as well as options to certain other JHL pipeline products. JHL will lead on development, registration and manufacturing, while Sanofi will be responsible for commercialization activities in China.

\section{Ignite Immunotherapy \& Pfizer}

The formation of Ignite Immunotherapy (CA, USA), a company focused on oncolytic vaccine design, was announced early in December. Pfizer is a strategic collaborative partner to Ignite, and is its lead investor (Pfizer will hold a 50\% equity investment in Ignite and has an exclusive option to acquire Ignite after the initial research program is completed). Ignite efforts are directed to the discovery and development of targeted, proprietary intravenous oncolytic virus vaccines for the immunotherapy of cancer. These agents could be developed for use in combination with other immunooncology agents such as checkpoint inhibitors, and could have the potential to cure patients with metastatic cancers [15]. Pfizer is one of several major players in the growing and successful field of immune-oncology and is clearly seeking to establish a unique space for itself.

\section{Bayer \& Versant Ventures - BlueRock Therapeutics}

Venture capital investment firm Versant Ventures this month also jointly invested (see paragraph on the merger of Northern Biologics and Mosaic Biomedicals earlier in this article) with Bayer AG (Leverkusen, Germany) to fund the creation of BlueRock Therapeutics. This new venture creates a regenerative medicine company planning to develop best-in-class induced pluripotent stem cell (iPSC) therapies, initially focusing on cardiovascular disease and neurodegenerative disorders. An initial program is aimed at regeneration of cardiac muscle in patients who have suffered a myocardial infarction and are at risk of secondary disease such as heart failure. This program is being advanced in collaboration with Torontobased McEwen Center for Regenerative Medicine and University Heath Network, where a cofounder of BlueRock and a world leader in stem cell biology, Dr Gordon Keller, is based. BlueRock also intends to collaborate with Toronto-based CCRM, a company that has developed technologies and expertise in GMP production of stem cell therapeutics [16].

\section{Novartis \& Conatus Pharmaceuticals}

Several companies are currently exploring innovative investigational therapies for fibrotic diseases and there is particular interest in non-alcoholic steatohepatitis as this disease has no currently approved treatments, and is expected to become the principal reason for liver transplantation by the end of the decade. Novartis announced it had taken an exclusive option, collaboration and license agreement with Conatus Pharmaceuticals Inc. (CA, USA) to develop new oral treatments for chronic liver diseases with the effort around joint development of emricasan [17]. Emricasan is an investigational, first-in-class oral, pan-caspase inhibitor (against apoptotic and inflammatory caspases) that has been the subject of multiple Phase II trials where it has demonstrated evidence of beneficial effect based on functional biomarkers of liver damage and inflammation as well as effects on caspase activity, which indicates the drug works as designed on to inhibit caspase-driven apoptosis of liver cells. Assessment of liver function overall suggested favorable trends relative to placebo. The drug has been well tolerated in these early Phase trials. Novartis envisages emricasan being administered as a single agent or in combination with Farnesoid X receptor agonists Novartis already has in development for the treatment of nonalcoholic steatohepatitis.

\section{Evotec \& Forge Therapeutics}

Forge Therapeutics (CA, USA), a start-up developing small molecule inhibitors that target metalloproteins, have discovered nonhydroxamate inhibitors of LpxC, which is not found in human cells or Grampositive bacteria but occurs across the Gram-negative bacteria. Historically, LpxC has been regarded as an attractive therapeutic target in developing agents for the therapy of serious Gram-negative infections, including drug resistant strains, but discovering good drugs has been hampered by finding suitable 
chemical starting points (beyond hydroxamic acids). The strategic alliance announced between Evotec, a drug discovery and development external partner to pharmaceutical and biotechnology companies, and Forge Therapeutics [18] provides for the resources to progress lead optimization of LpxC inhibitors with target of identifying a development candidate in the next couple of years. There is a clear unmet need to bring to the clinic new antibacterials with novel mechanisms of action to address the challenge of antibiotic resistance.

\section{Evotec \& Celgene}

A 5-year collaboration based on systematic screening of compounds using a unique iPSC platform was announced by Evotec and Celgene [19]. The industrialized iPSC screening platform at Evotec, developed over the last 5 years enabled through external research with Harvard University and an additional 10-year collaboration in the field of Huntington's disease, will be used to evaluate compounds in libraries at both companies. The aim is to identify potential disease-modifying agents for a broad range of neurodegenerative diseases. Initial focus will include amyotrophic lateral sclerosis, Alzheimer's disease, Parkinson's disease and other neurodegenerative disorders.

\section{Bristol-Myers Squibb \& Calithera Biosciences}

The ongoing evolution of immune-oncology, now regarded as a key treatment choice alongside surgery, radiotherapy and chemotherapy in the treatment of certain types of cancer continues to bring news of new agents and collaborations in the development of new agents in this area of therapy. South San Francisco-based Calithera Biosciences is engaged in discovery and development of small molecule drugs directed against tumor immunology and tumor metabolism targets. The company and Bristol-Myers Squibb announced [20] a clinical trial collaboration to evaluate Bristol-Myers Squibb's PD-1 checkpoint inhibitor nivolumab $\left(\right.$ Opdivo $\left.^{\circledR}\right)$ in combination with Calithera's orally administered glutaminase inhibitor, CB-839. CB-839 is currently in Phase I/II clinical studies, and is considered to act by starving tumor cells of glutamine, which may then reverse the resistance of tumors to checkpoint inhibitors by altering the immune-suppressive microenvironment within the tumor. The companies are looking to study the combination in patients with clear cell renal carcinoma (ccRCC) in a Phase II portion of an existing Calithera clinical study, aiming to achieve an improved and sustained efficacy in ccRCC patients where treatment with a PD-1 inhibitor is showing stable or still growing cancer.
Bristol-Myers Squibb \& PsiOxus Therapeutics In building a further collaboration involved in developing new approaches harnessing immunology for therapeutic intervention in oncology, Bristol-Myers Squibb announced its signing of an exclusive worldwide license agreement with Oxford, UK-based PsiOxus Therapeutics [21]. The agreement grants Bristol-Myers Squibb rights to NG-348 a preclinical stage 'armed' oncolytic virus, which will be developed for treatment of certain solid tumors. The approach developed for NG-348 by PsiOxus uses a modified virus, typically and adenovirus, which will selectively replicate within tumor cells but no in normal cells. Two integrated T-cell engaging protein are encoded within NG-348, a membrane anchored full-length human CD80 and a membrane anchored antibody fragment specific for the T-cell receptor $\mathrm{CD} 3$ protein. It is signaling by these two components, when expressed together on the surface of the NG-348 infected tumor cells, which leads to the accumulation of tumor infiltrating T-cells. Expression of the proteins is controlled by an endogenous virus major late promoter, which restricts the expression to cells permissive to virus infection, that is, tumor cells.

The use of the oncolytic viruses in this way may enhance the utility of checkpoint inhibitors. BristolMyers Squibb and PsiOxus already have an agreement in place from earlier in 2016 covering the nonarmed version of the oncolytic adenovirus, known as enadenotucirev.

\section{Other business news}

\section{GSK vaccines $R$ \& $D$ center}

GlaxoSmithKline (GSK, Brentford, UK) affirmed its commitment to vaccines as a therapeutic option within its $\mathrm{R} \& \mathrm{D}$ and commercial product portfolio when it opened its new global vaccines $\mathrm{R} \& \mathrm{D}$ centre in Rockville, MD USA, providing a facility for up to 450 scientist and support staff and creating up to 200 new jobs [22]. The newly opened site will complement the company's existing vaccine $\mathrm{R} \& \mathrm{D}$ centers in Rixensart, Belgium and Siena, Italy. GSK announced its intent to invest US $\$ 50$ over the next 2 years to continue to develop the site. The Rockville site was acquired by GSK in 2012 from Human Genome Sciences and projects at the site will include support for a shingles vaccine that GSK filed for regulatory approval with the US FDA in October 2016 as well as R\&D programs for RSV, Group B Streptococcus and dengue fever. 


\section{Product approvals \& other regulatory news}

\section{Nusinersen (SpinrazaTM, Biogen \& Ionis Pharmaceuticals)}

The FDA approved nusinersen injection for intrathecal administration, under a Priority Review, for the treatment of spinal muscular atrophy in pediatric and adult patients. Spinal muscular atrophy is a leading genetic cause of death in infants and toddlers that is marked by a progressive, debilitating muscle weakness which is caused by mutations in chromosome $5 \mathrm{q}$ and leads to $S M N$ protein deficiency. Nusinersen is an antisense oligonucleotide that alter the splicing of SMN2 premRNA to increase production of SMN protein. Filings are under review in other territories with possibility of approvals during 2017 [23].

Nusinersen was discovered by Ionis Pharmaceuticals (CA, USA). Biogen and Ionis established a broad strategic alliance in 2013 (Ionis Pharmaceuticals were known as Isis Pharmaceuticals at that time) that is focused on leveraging antisense technology to advance the treatment of neurological and neuromuscular disorders and around half a dozen programs are at various stages of development. Nusinersen is the first product from that alliance to advance through clinical trials to regulatory approval.

\section{Empagliflozin (Jardiance ${ }^{\circledR}$, Lilly)}

The FDA approved a new indication for empagliflozin (Jardiance, Eli Lilly and Company, IN, USA and Boehringer Ingelheim, NJ, USA) adding to the label for this previously approved SGLT2 inhibitor reduction in risk of cardiovascular death in adults with Type 2 diabetes and established cardiovascular disease [24]. The approval was based on a study that investigated the addition of empagliflozin (compared with placebo) to standard of care Type 2 diabetes and cardiovascular therapy in patients with Type 2 diabetes and established cardiovascular disease. The addition of empagliflozin significantly reduced the risk of the combined primary end point of cardiovascular death, nonfatal heart attack or nonfatal stroke, with the significant driver of the outcome being the reduction in cardiovascular death. Empagliflozin is currently the only oral Type 2 diabetes medicine approved by the FDA shown in a clinical trial to reduce the risk of cardiovascular death, although other SGLT2 inhibitors are in clinical trials exploring the same possible benefit for those agents.

\section{Pembrolizumab (Keytruda® ${ }^{\circledR}$, Merck)}

Merck (NJ, USA), also known as MSD outside the USA, announced that it had gained acceptance of and a priority review (with a due date of 15 March 2017) from the FDA for its sBLA application covering the immune-oncology compound pembrolizumab, a PD-1 checkpoint inhibitor, for the treatment of relapsed or refractory classical Hodgkin lymphoma. Classical Hodgkin lymphoma represents around 95\% of all Hodgkin lymphoma cases in developed countries. The approval of this application, if granted, will allow the use of pembrolizumab in patients who have relapsed after three or more prior lines of therapy [25].

\section{Axicabtagene ciloleucel (Kite Pharma)}

The advancement of CAR-T therapy into clinical practice represents an exciting aspect of therapy and its applications will likely, at least initially, in the treatment of disease where other options have failed or are not appropriate. Therefore, the news that Kite Pharma (CA, USA) had initiated a rolling submission with the FDA for a BLA for axicabtagene ciloleucel (also known as KTE-C19) was of some interest [26]. Axicabtagene ciloleucel is Kite's lead product candidate and is an investigational therapy that uses a patient's $T$ cells which are engineered to express a chimeric antigen receptor to target the antigen CD19, which is expressed on the cell surface of B-cell lymphomas and leukemias, thus directing the modified $\mathrm{T}$ cells to kill the cancer cells. The BLA is for the use of axicabtagene ciloleucel as a treatment for patients with relapsed or refractory aggressive B-cell non-Hodgkin lymphoma (NHL) and are ineligible for autologous stem cell transplant. Three subtypes of aggressive NHL were included in the clinical trials, chemorefractory diffuse large B-cell lymphoma, transformed follicular lymphoma (TFL) and primary mediastinal B-cell lymphoma. The clinical data were presented at ASH (see later section on clinical trials).

\section{Radiopaque embolic doxorubicin-eluting bead (DC bead LumiTM, BTG International)}

BTG International Canada, Inc. (Ottawa, Canada), part of the global BTG organization announced the commercial launch of DC Bead LUMITM in Canada, the first market to have this product available, although - subject to regulatory approvals - launches in additional markets worldwide are expected [27]. The product is a radiopaque embolic drug-eluting beads used in trans-arterial chemoembolization, where there is deliberate blocking of the blood supply to the tumor and local controlled delivery of chemotherapy. This product can be loaded with doxorubicin for the treatment of hepatocellular carcinoma, the commonest type of primary liver cancer, or loaded with irinotecan for the treatment of malignant colorectal cancer metastasized to the liver. DC Bead LUMITM is described as a next generation of DC $\mathrm{Bead}^{\circledR}$, the market-leading drugeluting embolic bead product [27]. 
Crisaborole (EucrisaTM, Pfizer)

Although this article noted earlier the acquisition of Ziarco by Novartis to enable progression through clinical trials of a novel oral therapy for eczema, it was Pfizer that this month announced the approval of the first new therapy for this condition for more than a decade in the form of crisaborole $2 \%$ ointment (Eucrisa ${ }^{\mathrm{TM}}$ ), a novel nonsteroidal topical treatment of mild-to-moderate eczema in patients 2 years of age and older [28]. It is estimated that $90 \%$ of patients with the condition have the mild to moderate form. Crisaborole is a PDE-4 inhibitor; overactive PDE- 4 is considered a contributor to the signs and symptoms of eczema. Crisaborole was acquired by Pfizer earlier in 2016 when it completed the acquisition of Anacor Pharmaceuticals, Inc.

\section{Everolimus (Votubia ${ }^{\circledR}$, Novartis) dispersible tablets}

A positive opinion was adopted by the EMA CHMP, a precursor of likely approval, for the dispersible tablet formulation of everolimus (Votubia) for treatment of refractory partial-onset seizures in patients with tuberous sclerosis complex (TSC) [29]. Everolimus dispersible tablets are already approved in the USA for TSC as Afinitor ${ }^{\circledR}$ dispersible tablets. TSC is a disease that can develop in the first year of life but can take years to develop before diagnosis can occur. It may cause nonmalignant tumors in various organs and some of the consequent resulting disorders are of a neurological nature. Seizures are the most common neurological manifestation in TSC and about $60 \%$ of patients become unresponsive to available therapies to control seizures. Everolimus dispersible tablets therefore address a currently poorly met need for these patients.

\section{Canakinumab (Ilaris ${ }^{\oplus}$, Novartis) for periodic fever syndromes}

Another new indication for an established Novartis product came in the positive CHMP recommendation for canakinumab (Ilaris), an IL-1 beta inhibitor, for the treatment of Periodic Fever Syndromes, a set of rare autoinflammatory diseases that mostly affect children and arise from noninfectious activation of the immune system [30]. Periodic Fever Syndromes can cause disabling and persistent fevers, accompanied by joint pain, muscle pain, swelling and rashes, with complications that can be life threatening. Existing therapies involve use of corticosteroids and nonsteroidal antiinflammatory drugs, but these approaches only help with symptoms rather than address underlying aspects of the syndromes.

A new $150 \mathrm{mg} / \mathrm{ml}$ solution formulation of canakinumab was used in clinical trials in treatment of three forms of periodic fever syndromes - tumor necrosis factor associated period syndrome, hyperimmunoglobulin D syndrome/mevalonate kinase deficiency and familial mediterranean fever and showed rapid and sustained disease control compared with placebo, therefore demonstrating its potential value in advancing the therapeutic option for patients with these syndromes [30]. Canakinumab is already approved in different territories for several inflammatory diseases where IL-1 Beta blockade is beneficial including Systemic Juvenile Idiopathic Arthritis, refractory gout arthritis and several subtypes of cryopyrin-associated periodic syndromes. Approved indications vary, depending on the individual country.

\section{Rucaparib (RubracaTM, Clovis Oncology)}

The approval of the first PARP inhibitor in the USA for the treatment of advanced ovarian cancer patients who carry the deleterious germline or somatic BRCA mutations and have already been treated with two or more chemotherapies was announced by Colorado, US-based Clovis Oncology [31]. The approval of rucaparib tablets (Rubraca ${ }^{\mathrm{TM}}$ ) as monotherapy, based on selection using an FDA-approved companion diagnostic, represents a real advance in the treatment of patients carrying a BRCA mutation and suffering from advanced disease that has already undergone several prior treatments. The approval was under the FDA's accelerated approval program so is based on less data than is usual for a typical approval, but this acceleration does bring forward an important approach to therapy quickly, but rupacarib's continued approval is contingent on confirmatory data from ongoing trials. Other PARP inhibitors are in development by a number of companies, including Abbvie and Tesaro, and olaparib from AstraZeneca is approved in some markets for specific indications.

\section{Clinical Trials}

\section{Enzalutamide (Xtandi ${ }^{\oplus}$, Pfizer \& Astellas)}

Pfizer and Astellas are continuing to collaborate on the development of enzalutamide (Xtandi), an orally administered androgen receptor inhibitor that acts to block multiple steps in androgen receptor signaling in tumor cells and is already approved in the USA for the treatment of patients with metastatic castration-resistant prostate cancer, or CRPC [32]. They jointly reported this month on the Phase 4 PLATO trial, where the efficacy and safety of continued treatment with enzaluatamide versus placebo added to abiraterone acetate and prednisone in patients with chemotherapy-naive metastatic CRPC whose prostatespecific antigen had progressed when previously treated with enzaluatamide. They reported the disappointing news that the trial failed to meet its primary end point 
of progression-free survival but committed to analyze the trial data further to help the two companies to continue to work together to focus on further helping men with metastatic CRPC.

\section{Osimertinib (Tagrisso ${ }^{\circledR}$, AstraZeneca)}

At the WCLC meeting in Vienna AstraZeneca presented data on osimertinib (Tagrisso) for the secondline treatment of patients with EGFR T790M mutation-positive locally advanced non-small cell lung cancer (NSCLC) in a Phase III trial [33]. More than a doubling of progression-free survival, improved by 5.7 months compared with that achieved with standard platinum-based chemotherapy was described, and the data suggest the potential for osimertinib to replace chemotherapy as the standard of care in the specific patient group, and be encouragingly promising in patients with CNS metastases, whose prognosis is often poor.

\section{Bosutinib (Bosulif®) (Avillion \& Pfizer)}

Collaborators Pfizer and Avillion LLP (London, UK) announced at ASH the results of a Phase III study demonstrating the superiority of their Bcr-Abl tyosine kinase/Src family kinases inhibitor bosutinib compared with imatinib as first0line therapy in patients with chronic phase Philadelphia chromosome positive myeloid leukemia [34]. Bosutinib (Bosulif) is currently approved in the USA and the EU for the treatment of adult patients with this disease who are resistant to or intolerant of prior therapy and this study will enable Pfizer to seek potential approval for the drug as first-line therapy.

\section{Glasdegib (PF-04449913, Pfizer)}

Also at ASH, Pfizer reported Phase II data for its investigational hedgehog pathway SMO inhibitor glasdegib added to low-dose cytarabine in acute myeloid leukemia and high-risk myelodysplasic syndrome in patients ineligible for intensive chemotherapy [35]. With the addition of glasdegib, median overall survival increased to 8.8 months relative to 4.9 months for low-dose cytarabine alone, thus suggesting the possibility of effective new treatment options in the treatment of this disease. Glasdegib is the first SMO inhibitor to demonstrate clinical benefit in this patient group.

\section{Crizanlizumab (SEG101, Novartis)}

Also at ASH, Novartis presented results of a Phase II study describing the reduction in the annual rate of sickle cell-related pain crises by $45.3 \%$ (relative to placebo) by the administration of a potential new diseasemodifying, preventive treatment crizanlinumab, also known as SEG101 (and formerly as SelG1) in patients with or without hydroxyurea treatment [36]. Hydroxyurea is a currently available therapy that is often not utilized because of concerns around potential adverse events and patient compliance. Crizanilumab is a humanized anti-P-selectin monoclonal antibody that blocks P-selectin on the surface of endothelial cells and the platelets in the blood vessels thus modulating its effect in the vaso-occlusive process associated with the blocking of blood flow by sickled erythrocytes.

Novartis gained the rights to crizanilumab when it acquired Selexys Pharmaceuticals Corporation, a company specializing in development of therapeutics in certain hematologic and inflammatory disorders, in November 2016.

\section{CTL019 (Novartis)}

In a further sharing of clinical trial findings at $\mathrm{ASH}$, Novartis presented data on an investigational CAR T-cell therapy, CTL019, in relapsed/refractory pediatric and young adult patients with B-cell acute lymphoblastic leukemia ( $\mathrm{r} / \mathrm{r}$ B-ALL) [37]. The trial, known as ELIANA, is the first pediatric global CAR T-cell registrational trial. Of the 50 subjects infused, 41 $(82 \%)$ achieved complete remission or complete remission with incomplete blood count recovery at 3 month post infusion. The estimated relapse-free rate among responders was $60 \% 6$ months post infusion and in those subjects who had achieved complete remission, no minimal residual disease was detected. Of the treated subjects, $48 \%$ experienced grade 3 or 4 cytokine release syndrome (CRS), a known complication of activation of the engineered cells in the patient's body and for which there was an active management approach as part of the trial. Novartis expects to apply for regulatory approval for CTL019 in both the USA and the EU during 2017.

\section{Ceritinib (Zykadia ${ }^{\circledR}$, Novartis)}

Novartis was also busy sharing clinical trial data at the WCLC meeting, where they reported on the treatment of ALK+ NSCLC with ceritinib (Zykadia) a selective inhibitor of ALK, a gene that can fuse with others leading to an abnormal fusion protein that promotes the growth of certain tumors including NSCLC [38]. Certinib is already approved in the US an EU for the treatment of patients with ALK+ NSCLC previously treated with crizotinib whose disease continues to progress. The new trial data, from the ASCEND- 4 trial, showed that patients given first-line treatment with ceritinib doubled their progression-free survival time relative to patients given first-line standard chemotherapy. Novartis also shared early results of a fed versus fasted bioavailability study for ceritinib, which showed that compared with dosing $750 \mathrm{mg}$ in the 
fasted state as currently, indicated dosing with a low fat meal led to comparable pharmacokinetics for a 450 $\mathrm{mg}$ dose and an increase of $25 \%$ in bioavailability for a $600 \mathrm{mg}$ dose.

\section{NEOD001 (Prothena Biosciences)}

Results from Phase I/Phase II studies investigating the treatment of immunoglobulin light-chain amyloidosis with an antibody, NEO001 developed by Prothena Biosciences (CA, USA), were presented at the ASH meeting [39]. Amyloidosis is a rare and life-threatening condition which manifests as deposition of toxic amyloid protein in various organs including gastrointestinal tract, heart, kidney, liver and disrupts their function. Treatment with bortezomid, a proteasome inhibitor, can kill the abnormal cells producing the abnormal proteins but this does not eliminate already laid-down amyloid. NEO001 targets AL amyloid specifically, and is thought to neutralize soluble amyloid and induce clearance of insoluble deposited fibrils. Monthly infusion of NEO001, after completion of standard treatment, resulted in improvement of peripheral neuropathy and, based on biomarker responses, $53 \%$ of patients showed improvement in cardiac functional biomarkers and $64 \%$ of those treated showed improvement in renal function biomarkers. Phase IIb and Phase III studies of NEO001 are ongoing.

\section{Obinutuzumab (Gazyva ${ }^{\circledR} /$ Gazyvaro $^{\circledR}$, Roche)}

A Phase III study, known as GALLIUM, of obinotuzumab (Gazyva/Gazyvaro), a monoclonal antibody therapeutic from Roche that is already approved in a number of territories for use with chlorambucil for the treatment of chronic lymphocytic leukemia was described at ASH showing that when used with chemotherapy it reduced the risk of disease worsening or death by $34 \%$ compared with rituxan-based standard of care (rituxan plus chemotherapy followed by rituxan alone) in follicular lymphoma (FL), the most common slow-growing form of NHL [40]. This study, a third positive Phase III study in lymphocytic lymphoma will form the basis of an upcoming regulatory approval submission by Roche for this therapy in the specific indication studied in the GALLIUM trial.

\section{SPK-9001 (Spark Therapeutics \& Pfizer)}

Results from a Phase 1/Phase II study of a gene therapy treatment for hemophilia B were shared by Pfizer and Spark Therapeutics (PA, USA) at the ASH meeting [41]. The investigational product SPK-9001, based on adeno-associated viral vectors, had been given to nine subjects with hemophilia $B$ as a single dose of 5 $\times 10^{11}$ vector genomes per kilogram body weight and subjects were followed for up to 1 year post treatment.
Over the nine subjects the total factor IX concentrates consumption over a cumulative 1650 patient days following vector administration was reduced by 1.13 million international units relative to their usage in the year before treatment with SPK-9001. OF the nine subjects two demonstrated symptoms of an immune response to the vector capsid resulting in a decline of factor IX activity level, in both cases this was managed with corticosteroids and neither subject experienced bleeds or required factor IX concentrate infusion. It appeared that the immune response is transient and can be arrested by corticosteroid treatment. One subject had reached one full year post SPK-9001 infusion without any requirement for factor IX concentrate infusion and without having any bleeds. Based on the experience of this study, particularly managing the immune response, Spark and Pfizer will be able to better design other upcoming hemophilia studies on the route to gathering sufficient clinical data for regulatory submissions for this gene therapy.

\section{Ibrutinib (Imbruvica $\left.{ }^{\circledR}\right)$ AbbVie}

AbbVie (IL, USA) presented two sets of clinical studies of ibrutinib (Imbruvica) at the ASH meeting. Ibrutinib is a first-in-class BTK inhibitor jointly developed and commercialized by Pharmacyclics, an AbbVie company, and Janssen Biotech, Inc (PA, USA).

One report was on a 5-year analysis and long-term follow-up in chronic lymphocytic leukemia/small lymphocytic leukemia (CLL/SLL), a form of lymphocytic lymphoma that is predominantly a disease of the elderly (median age at diagnosis is 71) and the second report was around two trials on its use in combination with rituximab and lenalidomide in the treatment of two common types of NHL - diffuse large B-cell lymphoma (DLBCL), the most common form of aggressive lymphoma and FL $[42,43]$. About a third of FL cases develop into the more aggressive DLBCL.

In the study in CLL/SLL, nearly nine out of ten patients $(89 \%)$ achieved a complete or partial response at the 5-year analysis. Almost a third of patients (29\%) who received single agent ibrutinib as first treatment for their disease achieved complete response; patients lived without disease progression longer when treatment was started earlier in the course of the disease. AbbVie indicated that this trial data along with other ibrutinib studies offer evidence that extended treatment with ibrutinib may benefit a wide range of CLL/ SLL patients, including those with genetic mutations that may make their disease difficult to treat with other agents.

For the trial in DLBCL, while existing therapies can be effective and cure over half of the patients with DLBCL, patients with relapsed or refractory disease 
have a need for new treatments, as less than a quarter of these patients then respond to further treatment with established therapies for the specific patient group. Preliminary efficacy results with the combination treatment encouragingly showed the highest response rate in the patients with the worst prognosis and this finding will now lead to a Phase II study of the combination therapy in paints with this poorly met medical need.

In a separate Phase II study of ibrutinib with rituximab, AbbVie reported favorable outcomes for patients with previously untreated $\mathrm{FL}$, with $35 \%$ of patients showing a complete response and claimed the addition of ibrutinib to rituximab provides enhanced outcomes over use of rituximab alone in this disease.

\section{AMT-060 (uniQure)}

The gene therapy product from Spark Therapeutics for the treatment of hemophilia B described earlier in this article is in competition with another proprietary gene therapy for the same indication. Amsterdam-based uniQure presented data from their investigational product, AMT-060, which is being developed for patients with severe hemophilia B at the ASH meeting [44]. With follow-up data for up to 2 weeks in a low-dose cohort and up to 31 weeks for a second-dose cohort, uniQure described substantial improvement in disease state for the second-dose cohort, including discontinuation of Factor IX infusions in four of five subjects treated. For the low-dose cohort they described the patients as having robust, constant and clinically meaningful levels of Factor IX activity for up to 52 weeks post treatment. Corticosteroid treatment to deal with adverse events was required for two of the five subjects in the second-dose cohort and one of the five in the low-dose cohort.

\section{Axicabtagene ciloleucel (Kite Pharma)}

Kite Pharma's CAR T-cell therapy axicabtagene ciloleucel, also referred to as KTE-C19, was reported on at the ASH meeting based on data from a trial, ZUMA1 , in patients with a chemorefractory aggressive NHL, either DLBCL, primary mediastinal B-cell lymphoma or TFL [45]. CAR T-cell product manufacture was successful in all but one of the 111 patients enrolled in the ZUMA-1 trial and 101 subjects were treated. In patients with not less than 3 month follow-up post dosing, compete response was observed in $52 \%$ of patients in the trial, with this rising to $73 \%$ complete response for the TFL/primary mediastinal B-cell lymphoma group, which for a group of patients in real need of more effective therapies is very encouraging. Additional data are expected to be made available in the first quarter of 2017. Typical of CAR T-cell therapy there were CRS and neurological (NE) adverse events (13\% and $29 \%$ of subjects showing grade 3 or greater) but there were no cases of cerebral edema. Significant adverse events other than CRS and NE occurred in up to $63 \%$ of patients treated and three patients died from treatment emergent adverse events. However, this is a patient group in very real need of new and effective therapies.

\section{Ribociclib (LEE011, Novartis)}

Ribociclib, LEE011, is an investigational selective cyclin-dependent kinase inhibitor that targets CDK4 and CDK6, which if overactivated can enable cancer cells to grow divide too quickly and thus might help manage the uncontrol edgrowth of some cancer cells. It is being developed by Novartis under a research agreement with Astex Pharmaceuticals. Novartis described the outcome of additional analyses of a Phase III trial in selected patient subgroups with hormone receptor positive, $\mathrm{HR}^{+} / \mathrm{HER} 2^{-}$advanced or metastatic breast cancer, including newly diagnosed postmenopausal women, those with liver and lung metastases and those with bone-only disease where ribociclib was added to letrozole. The data suggested the utility of the combination as fist-line therapy across all patient subgroups, and suggests it may be a useful treatment for the postmenopausal patients who are often diagnosed with advanced breast cancer that has already metastasized and are in much need of treatment that can extend time until disease progression. Novartis has several Phase III trials of ribociclib underway [46].

\section{Paclitaxel protein-bound particles injectable suspension (Abraxane ${ }^{\oplus}$, Celgene)}

Metastatic triple negative breast cancer remains one of the most challenging forms of breast cancer, representing a major unmet need and where there is considerable ongoing effort to find more effective treatment options for patients. Celgene presented the result of their tnAcity Phase II trial to evaluate the use of paclitaxel protein-bound particles injectable suspension (Abraxane) as a component of first-line treatment of metastatic triple negative breast cancer. The trial found that a weekly combination regime of paclitaxel protein-bound particles injectable suspension with carboplatin had a significantly longer progressionfree survival compared with paclitaxel protein-bound particles injectable suspension with gemcitabine or carboplatin with gemcitabine. However, the trial outcome was considered in the light of the rapidly changing breast cancer treatment landscape, notably to increasing focus on the utility of immune-oncology treatments, and in their press release around the study results, Celgene stated that they had determined 
not to move forward with a Phase III portion of the tnAcity trial. Instead they plan to focus on the use of paclitaxel protein-bound particles injectable suspension in combination with immunotherapy agents but would be using the completed research from tnAcity to inform ongoing and future research in breast cancer for patients with high unmet needs [47].

\section{Aducanumab (BIIB037, Biogen)}

The amyloid hypothesis for the development and progression of Alzheimer's disease remains a focus of ongoing research into treatment of the disease despite several high profile clinical trial failures of agents that engage in amyloid processing. An update on data from a Phase Ib study of the investigational Alzheimer's disease treatment aducanumab (BIIB037) in patients with prodromal or mild Alzheimer's disease, including results from a 12-month long-term extension was presented by Biogen at Clinical Trials on Alzheimer's Disease [48]. Aducanumab is a human recombinant monoclonal antibody derived from B-cells collected from healthy elderly subjects with no signs of cognitive impairment or from cognitively impaired elderly subjects exhibiting unusually slow cognitive decline, which was licensed by Biogen from Neurimmune. It binds aggregated but not monomeric forms of beta amyloid. In mouse models of Alzheimer's disease it has been shown to remove amyloid plaques form the brain and the possibility is therefore it could offer therapeutic benefit by slowing or stopping amyloid pathology in Alzheimer's disease patients at the preclinical phase of the disease by clearing amyloid plaques from the brain. The newly shared data showed that in patients previously on low-dose therapy switched to high-dose therapy and patients originally on high-dose therapy maintained on that all showed decreased amyloid plaque burden and changes from baseline on other clinical end points. No new safety signals form earlier analyses were observed. Earlier safety concerns were the dose-dependent increased incidence of vasogenic edema linked to amyloid-related imaging abnormalities (ARIA-E) in carriers of the apolipoprotein $\mathrm{E} 4$ allele (which is associated with greater risk of developing Alzheimer's disease). It was reported that by gradually raising the dose then the incidence of ARIA-E was reduced. On the basis of these promising early Phase results, Biogen have already initiated Phase III trials.

\section{Volanesorsen (Ionis Pharmaceuticals)}

Volanesorsen is an antisense drug which can decrease plasma triglyceride levels by directly targeting apolipoprotein production in the liver. It is under development by Akcea Therapeutics (Cambridge, USA), a wholly-owned subsidiary of Ionis Pharmaceuticals. Its therapeutic use in the treatment of severe hypertriglyceridemias (triglyceride levels greater than $500 \mathrm{mg} / \mathrm{dl}$ ), including the rare metabolic disorders familial chylomicronemia syndrome (FCS) and familial partial lipodystrophy is being explored in the COMPASS Phase III trial. The two companies announced positive results from the COMPASS study which showed a mean reduction in triglycerides of $71.2 \%$ after 13 weeks treatment (vs $0.9 \%$ for placebo and this was sustained through the 26 weeks treatment period). For the FCS patient subgroup the reduction was $73 \%$ reduction from baseline versus an increase of $70 \%$ on placebo [49]. Current therapies for extremely high triglycerides are regarded as inadequate, leaving patients with significant mortality and morbidity risk. COMPASS is one of four global trials of volanesorsen in progress, with data for first regulatory filings for FCS being available in early 2017, and additional filings for additional indications planned thereafter.

\section{Cabotegravir long acting injection (Viiv)}

ViiV Healthcare (Brentford, UK), which specializes in the development of new therapies for the treatment of HIV infection announced the start of Phase III trials for its long-acting injectable HIV integrase inhibitor cabotegravir as prevention of HIV infection in subjects at high risk of infection with HIV. Long-acting cabotegravir dosed every 2 months will be compared with daily oral Truvada ${ }^{\circledR}$, which is the only currently licensed pre-exposure prophylaxis agent for those at high risk of HIV infection. The trial will determine if the new injectable option may offer adherence to therapy benefits over a daily oral medication, with adherence to therapy potentially being important in better assuring prevention of disease [50].

\section{Dolutegravir Tivicay ${ }^{\circledR}$, Viiv) with rilpivirine (Edurant ${ }^{\oplus}$, Janssen) $^{\circ}$}

Also from ViiV this month came the announcement of positive results from the first Phase III studies of a two-drug HIV treatment regimen in patients switched to the new therapy from, and showing effective viral suppression, on the more usual three of four-drug regimen (integrase inhibitor-, non-nucleoside reverse transcriptase inhibitor or boosted protease inhibitor-based therapy). The two-drug regimen employed ViiV's dolutegravir (Tivicay) with rilpivirine (Edurant) from Janssen Sciences Ireland (part of Johnson and Johnson) and the study met its primary end point of noninferiority of switching to the two-drug regimen against remaining on the three- or four-drug regimen based on the proportion of subjects having plasma HIV-1 RNA of $<50$ copies $/ \mathrm{ml}$. The partnership on the investigate of the potential of combining the two agents was established in June 2014 [51]. 
Nivolumab (Opdivo, Bristol-Myers Squibb) with ipilimumab (Yervoy ${ }^{\oplus}$, Bristol-Myers Squibb)

Bristol-Myers Squibb provided updates on clinical trials of treatment of small cell and non-small-cell lung cancer (SCLC and NSCLC, respectively) with a combination of its immune PD-1 checkpoint inhibitor nivolumab (Opdivo) and its anti-CTLA4 antibody drug ipilimumab (Yervoy) at WCLC [52,53]. In a Phase I/Phase II study in recurrent SCLC the trail primary objective was achieved in $25 \%$ of subjects treated with the combination and in $11 \%$ of subjects treated with nivolumab alone. Of the 61 subjects treated with the combination therapy in the trial, three showed a complete response. SCLC is a rapidly progressive disease, most patients relapsing within a year of diagnosis, and now new treatment options have emerged to help these patients in the last 30 years. It was reported that the responses in this trial for this group of patients are indicative of promising survival and indeed 30\% of subjects treated with the combination were alive at 2 years post start of the treatment [52].

In the Phase $\mathrm{Ib}$ trail of the combination therapy of advanced NSCLC, Bristol-Myers Squibb announced encouraging progression-free survival, with a near doubling or response rates with the combination relative to prior data on nivolumab alone in both PD-L1 expressors and nonexpressors. PD-L1 status is considered by some to be a biomarker for selecting patients for treatment with PD-1 immune checkpoint inhibitors. The data are suggested as indicative of the potential of the combination for evaluation as a first-line treatment of advanced lung cancer [53].

\section{Pimavanserin (Nuplazid ${ }^{\oplus}$, Acadia)}

Pimavanserin (Nuplazid) has been under development by Acadia Pharmaceuticals (CA, USA) for several unmet needs in CNS disorders and announced positive Phase II results from trials in the treatment of Alzheimer's disease psychosis [54] and as adjunctive treatment for patients with major depressive disorder [55].

Pimavanserin is a selective serotonin inverse agonist preferentially targeting $5-\mathrm{HT}_{2 \mathrm{~A}}$ receptors, and for the psychosis indication is novel as it has a different mechanism of action compared with currently marketed psychotics, including the atypical antipsychotics which have been associated with worsening of cognitive function when used to treat Alzheimer's patients. It already has FDA approval for the treatment of hallucinations and delusions associated with Parkinson's disease psychosis. It showed a statistically significant treatment improvement in psychosis at week 6 of treatment relative to placebo and over 12 weeks of therapy there was no impairment of cognition, based on similarity of outcomes to the placebo group. Further analyses of the data and future development plans beyond Phase II will be presented by Acadia in due course [54].

For the major depressive disorder indication, the announcement from Acadia covered forward plans to initiate a trial in patients with this disease where they have had inadequate response to first-line therapies. These patients are in need of treatment to improve clinical outcomes, as the majority of people who have this disease do not respond to initial antidepressant therapy and it is a leading cause of disability in the USA for the 15-44 years' age group [55].

\section{Larotrectinib (LOXO-101, Loxo Oncology)}

Loxo Oncology (CA, USA) described the Phase I trial data for its TRK inhibitor larotrectinib (LOXO-101) at the European Society for Medical Oncology Asia Congress [56] and announced its forward plan for the compound in terms of seeking FDA approval [57]. The genes that encode for TRKs can become abnormally fused to other genes. This results in signaling that leads to cancer at many sites in the body. In the Phase I trial of the 59 enrolled patients (patients who were not responding to standard therapy) eight had refractory solid tumors harboring TRK fusions. Of those eight, seven were evaluable and six of those achieved a confirmed partial response. All responders remained in response through ongoing treatment. The path forward to US regulatory filing is based on the fact that this is a targeted therapy drug so has a small database size based on precedents already set for targeted therapies and revolves around complete enrolling for primary efficacy analyze in early 2017. Reportable top-line data are expected in second half of 2017 and US NDA filing in late 2017 or early 2018. An EU MAA filing could occur in 2018.

\section{Brentuximab vedotin (Adcetris ${ }^{\oplus}$, Seattle Genetics)}

Brentuximab vedotin (Adcetris) is an antibody-drug conjugate (ADC) based on an anti-CD30 monoclonal antibody linked to monomethyl auristatin E, a very potent microtubule polymerization inhibitor, using proprietary conjugation technology. The conjugation technology and the ADC were developed by Bothell, USA-based Seattle Genetics; monomethyl auristatin E is a synthetic agent based on the marine organism natural product dolastatin. Seattle Genetics has around a dozen ADCs at various stages of clinical development and has ADC technology collaborations with at least nine partners including among others, AbbVie, Astellas, Bayer Genentech GSK and Pfizer.

As Adcetris, brentuximab vedotin is already approved for the treatment of classical Hodgkin lymphoma and relapsed systemic anaplastic large cell lymphoma, in 
65 countries including the USA (where it was the first product approved for classical Hodgkin lymphoma in more than 30 years) Canada, the EU and Japan. The specifics of the indications it is approved for, and how it is approved for, use may vary from country to country. Systemic anaplastic large cell lymphoma is an aggressive type of T-cell NHL. Seattle Genetics have been developing this agent in coloration with Takeda Pharmaceutical Company Limited, who hold commercial rights for all territories except USA and Canada where Seattle Genetics retain rights.

The collaborators announced positive Phase III data from a trial (ALKANZA) in a new indication, CTCL [58]. This disease is a form of NHL that primarily involves the skin, and where in $50 \%$ of patients the skin lesions express CD-30. The most common subtypes are mycosis fungoides and primary cutaneous anaplastic large cell lymphoma. In subjects with CTCL, brentuximab vedotin therapy showed improvements in measures of efficacy over standard of care therapy (including methotrexate and bexarotene) with an objective response rate lasting at least 4 months in $56.3 \%$ of ADC treated subjects (where the complete response rate was $15.6 \%$ ) versus $12.5 \%$ in the control arm (complete response rate 16\%). Median progression-free survival was 16.7 months for the brentuximab vedotin group, versus 3.5 months for the control group. Regulatory filing for approval for use in CTCL are expected in early 2017.

\section{References}

1 Actelion (SIX: ATLN) confirmed today that it has been approached by Johnson \& Johnson about a possible transaction.

www.actelion.com/en/our-company/news-and-events. page? newsId $=2059449$

2 Johnson \& Johnson (NYSE:JNJ) today confirmed it has ended discussions with Actelion Pharmaceuticals Ltd. www.jnj.com/media-center/press-releases/johnson-johnsonannounces-it-has-ended-discussions-with-actelion

3 Actelion update on strategic traction discussions. wwwl.actelion.com/en/investors/news-archive/index. page? newsId $=2064901$

4 Sanofi said to be in advanced talks to acquire Actelion. www.bloomberg.com/news/articles/2016-12-16/sanofi-saidto-be-in-advanced-talks-to-buy-drugmaker-actelion

5 Actelion and Johnson and Johnson enter into exclusive discussions. www.actelion.com/en/our-company/news-and-events. page? newsId $=2067118$

6 Johnson \& Johnson Confirms Exclusive Negotiations with Actelion.

www.jnj.com/media-center/press-releases/johnson-johnson-
Seattle Genetics, in collaboration with Bristol-Myers Squibb, are also pursuing use of brentuximab vedotin in combination with the immune checkpoint inhibitor nivolumab (Opdivo) in the treatment of relapsed or refractory classical Hodgkin lymphoma and the two companies announced preliminary data [59] from a Phase I/Phase II trial of the combination therapy that demonstrated it to be a promising approach in subjects who had failed during first-line therapy. In the patient cohort studied, $90 \%$ of evaluable patients $(n=29)$ had an objective response, $62 \%$ had a complete metabolic response and $28 \%$ had a partial metabolic response. The results were considered as supporting further exploration of the combination therapy, including as frontline treatment in certain patient groups [59].

\section{Financial \& competing interests disclosure}

The author is a former employee, and a current shareholder, of Bristol-Myers Squibb, which has active research programs and marketed medicines in some of the therapeutic areas covered in this article and some products are mentioned by name. The author has no other relevant affiliations or financial involvement with any organization or entity with a financial interest in or financial conflict with the subject matter or materials discussed in the manuscript. Apart from as noted, this includes employment, consultancies, honoraria, stock ownership or options, expert testimony, grants or patents received or pending, or royalties. No writing assistance was utilized in the production of this manuscript.

confirms-exclusive-negotiations-with-actelion

$7 \quad$ Novartis bolsters innovative dermatology portfolio through acquisition of Ziarco Group Limited. www.novartis.com/news/media-releases/novartis-bolstersinnovative-dermatology-portfolio-through-acquisition-ziarco

8 Encore Vision announces successful Phase I - II study of topical EV06 for the treatment of presbyopia.

http://encorevisioninc.com/2016/05/05/encore-visionannounces-successful-phase-I-ii-study-topical-ev06treatment-presbyopia

9 Novartis bolsters ophthalmology pipeline through acquisition of Encore Vision, Inc. www.novartis.com/news/media-releases/novartis-bolstersophthalmology-pipeline-through-acquisition-encore-visioninc

10 Northern Biologics, Mosaic Biomedicals execute transformative deal.

http://northernbiologics.com/wp-content/uploads/2015/12/ Northern-Mosaic-PR-FINAL.pdf

11 Bicycle Therapeutics. www.bicycletherapeutics.com

12 IBM and Pfizer to accelerate immuno-oncology research with Watson for Drug Discovery. 
www.ibm.com/blogs/watson-health/ibm-and-pfizer-toaccelerate-immuno-oncology-research-with-watson-fordrug-discovery/www.pfizer.com/news/press-release/ press-release-detail/ibm_and_pfizer_to_accelerate_immuno_ oncology_research_with_watson_for_drug_discovery

13 Cerevance launched with new technology, drug portfolio and $\$ 36 \mathrm{M}$ in cash to tackle brain diseases. www.takeda.com/news/2016/20161202_7638.html Sanofi and JHL Biotech announce strategic biologics alliance in China. www.jhlbiotech.com/news.php?ckid=7

15 Pioneers in oncolytic virus and gene therapy fields announce formation of IGNITE Immunotherapy Inc., a company focused on oncolytic cancer vaccine discovery and development.

http://finance.yahoo.com/news/pioneersoncolytic-virus-gene-therapy-130000847.html; ylt=AwrC0CMW0k5YhjcASBzQtDMD;_ylu=X3oDMTBy OHZyb21tBGNvbG8DYmYxBHBvcwMxBHZ0aWQDBH NlYwNzcg-therapy company BlueRock Therapeutics with USD225 million Series A financing. www.news.bayer.com/baynews/baynews.nsf/id/BayerVersant-Ventures-Join-Forces-Launch-Stem-Cell-TherapyCompany-BlueRock-Therapeutics-USD

17 Novartis announces exclusive option, collaboration and license agreement with Conatus to develop new oral treatments for chronic liver diseases.

www.novartis.com/news/media-releases/novartis-announcesexclusive-option-collaboration-and-license-agreementconatus

18 Evotec and Forge Therapeutics form strategic 'superbug' alliance.

www.evotec.com/archive/en/Press-releases/2016

Evotec-and-Forge-Therapeutics-form-strategic-superbugalliance $/ 2902 / 1$

19 Evotec and Celgene enter into drug discovery collaboration for neurodegenerative diseases. www.evotec.com/archive/en/Press-releases/2016/Evotecand-Celgene-enter-into-drug-discovery-collaboration-forneurodegenerative-diseases/2908/1

20 Bristol-Myers Squibb and Calithera Biosciences announce clinical collaboration to evaluate Opdivo (nivolumab) in combination with CB-839 in clear cell renal cell carcinoma. http://news.bms.com/press-release/partnering-news/bristolmyers-squibb-and-calithera-biosciences-announce-clinicalcolla

21 Bristol-Myers Squibb signs exclusive worldwide license agreement with PsiOxus Therapeutics for NG-348, an 'armed' oncolytic virus to address solid tumors. http://news.bms.com/press-release/partnering-news/bristolmyers-squibb-signs-exclusive-worldwide-license-agreementpsiox

22 US FDA approves Biogen's SPINRAZATM (nusinersen), the first treatment for spinal muscular atrophy. http://newsroom.biogen.com/press-release/ neurodegenerative-diseases/us-fda-approves-biogensspinraza-nusinersen-first-treatment
23 GSK opens new global vaccines R\&D centre in Rockville, MD, USA.

www.gsk.com/en-gb/media/press-releases/2016/gsk-opensnew-global-vaccines-randd-center-in-rockville-md-usa

24 US FDA approves Jardiance ${ }^{\circledR}$ (empagliflozin) tablets to reduce the risk of cardiovascular death in adults with type 2 diabetes and established cardiovascular disease.

https://investor.lilly.com/releasedetail.

cfm?ReleaseID $=1002600$

25 FDA grants priority review to supplemental Biologics License Application (sBLA) for Merck's KEYTRUDA ${ }^{\circledR}$ (pembrolizumab) in relapsed or refractory classical Hodgkin lymphoma.

www.mercknewsroom.com/news-release/oncologynewsroom/fda-grants-priority-review-supplemental-biologicslicense-application

26 Kite Pharma initiates rolling submission of US Biologics License Application (BLA) for KTE-C19, its investigational anti-CD19 CAR-T therapy, for the treatment of patients with relapsed/refractory aggressive B-cell non-Hodgkin lymphoma (NHL).

http://ir.kitepharma.com/releasedetail. cfm?ReleaseID $=1002522$

27 Canada first to receive new treatment option for liver cancer patients.

www.btgplc.com/media/press-releases/canada-first-toreceive-new-treatment-option-for-liver-cancer-patients

28 Eucrisa approved for patients 2 years of age and older. www.pfizer.com/news/press-release/press-release-detail/ pfizer_receives_fda_approval_for_eucrisa_crisaborole_a novel_non_steroidal_topical_ointment_for_mild_to_ moderate_atopic_dermatitis_eczema

29 Novartis drug Votubia ${ }^{\circledR}$ recommended by CHMP for EU approval to treat refractory partial-onset seizures in patients with TSC.

www.novartis.com/news/media-releases/novartis-drugvotubiar-recommended-chmp-eu-approval-treat-refractorypartial

30 Novartis receives positive CHMP recommendation for Ilaris ${ }^{\circledR}$ to treat rare diseases in adults and children called Periodic Fever Syndromes. www.novartis.com/news/media-releases/novartis-receivespositive-chmp-recommendation-ilarisr-treat-rare-diseases

31 Clovis Oncology Announces FDA Accelerated Approval of RUBRACA ${ }^{\mathrm{TM}}$ (rucaparib) for the Monotherapy Treatment of Advanced Ovarian Cancer in Women with Deleterious Germline or Somatic BRCA Mutations Treated with Two or More Chemotherapies.

http://phx.corporate-ir.net/phoenix. zhtml?c=247187\&p=irol-newsArticle_Print $\& I D=2230642$

32 Pfizer and Astellas announce top-line results from Phase 4 PLATO trial of XTANDI ${ }^{\circledR}$ (enzalutamide) capsules in patients with metastatic castration-resistant prostate cancer. www.pfizer.com/news/press-release/press-release-detail/ pfizer_and_astellas_announce_top_line_results_from_ phase_4_plato_trial_of_xtandi_enzalutamide_capsules_ in_patients_with_metastatic_castration_resistant_prostate_ cancer 
33 Tagrisso demonstrates superiority over chemotherapy in EGFR T790M mutation-positive non-small cell lung cancer. www.astrazeneca.com/media-centre/press-releases/2016/ tagrisso-demonstrates-superiority-over-chemotherapyin-egfr-t790m-mutation-positive-non-small-cell-lungcancer-06122016.html

34 Pfizer and Avillion announce positive top-line results for Phase III BFORE study of BOSULIF for first-line treatment of Philadelphia chromosome positive chronic myeloid leukemia. www.pfizer.com/news/press-release/press-release-detail/ pfizer_and_avillion_announce_positive_top_line_results_ for_phase_3_bfore_study_of_bosulif_for_first_line_ treatment_of_philadelphia_chromosome_positive_chronic_ myeloid_leukemia

35 New Phase II results show investigational compound glasdegib improved overall survival in acute myeloid leukemia and high-risk myelodysplastic syndrome. www.pfizer.com/news/press-release/press-release-detail/ new_phase_2_results_show_investigational_compound_ glasdegib_improved_overall_survival_in_acute_myeloid_ leukemia_and_high_risk_myelodysplastic_syndrome

36 Novartis SEG101 (crizanlizumab, formerly SelG1) significantly reduces frequency of sickle cell pain crises in Phase II study. www.novartis.com/news/media-releases/novartis-seg101crizanlizumab-formerly-selg1-significantly-reduces-frequency

37 Novartis presents results from first global registration trial of CTL019 in pediatric and young adult patients with $r / r$ B-ALL. www.novartis.com/news/media-releases/novartis-presentsresults-first-global-registration-trial-ctl019-pediatric-and

38 Novartis announces Zykadia ${ }^{\circledR}$ first-line study results showing 16.6 month progression-free survival in patients with ALK+ advanced NSCLC.

www.novartis.com/news/media-releases/novartis-datashows-treatment-free-remission-rates-are-consistentlyabove- 50

39 Prothena Archived Events, ASH presentations on NEOD001. http://ir.prothena.com/events.cfm

40 Roche's Gazyva/Gazyvaro helped people with previously untreated follicular lymphoma live significantly longer without their disease worsening compared to MabThera/ Rituxan.

www.roche.com/media/store/releases/med-cor-2016-12-05. htm

41 Spark Therapeutics and Pfizer present updated preliminary data from hemophilia B Phase $1 / 2$ trial suggesting consistent and sustained levels of Factor IX activity at annual ASH meeting.

http://ir.sparktx.com/phoenix.zhtml?c=253900\&p=irol-new sArticle \&cat $=$ news $\&$ id $=2227316$

42 Imbruvica ${ }^{\circledR}$ (ibrutinib) chronic lymphocytic leukemia/ small lymphocytic lymphoma 5-Year analysis and long-term RESONATE-2 follow-up data at ASH 2016 show lasting efficacy.

https://news.abbvie.com/news/imbruvica-ibrutinib-chroniclymphocytic-leukemiasmall-lymphocytic-lymphoma-5-yearanalysis-and-long-term-resonate-2-follow-up-data-at-ash2016-show-lasting-efficacy.htm
43 Favorable response rates suggest promise of ibrutinib $\left(\right.$ Imbruvica ${ }^{\circledR}$ ) combination therapy in two common types of non-Hodgkin's lymphoma.

https://news.abbvie.com/news/favorable-response-ratessuggest-promise-ibrutinib-imbruvica-combination-therapyin-two-common-types-non-hodgkins-lymphoma.htm

44 uniQure announces first clinical data from second dose cohort of AMT-060 in ongoing Phase I/II trial. http://uniqure.com/investors-newsroom/press-releases.php

45 Kite Pharma presents results of multi-center pivotal ZUMA1 trial of axicabtagene ciloleucel (KTE-C19) in aggressive non-Hodgkin lymphoma as late-breaking abstract at Annual Meeting of American Society of Hematology. http://ir.kitepharma.com/releasedetail. cfm?ReleaseID = 1002918

46 Novartis LEE011 (ribociclib) plus letrozole analyses show superior PFS across broad spectrum of patients in first-line HR+/HER2- advanced breast cancer versus letrozole. www.novartis.com/news/media-releases/novartis-lee011ribociclib-plus-letrozole-analyses-show-superior-pfs-across

47 tnAcity data presented at SABCS evaluates the investigational use of Abraxane ${ }^{\circledR}$ as first-line treatment of metastatic triple negative breast cancer. http://ir.celgene.com/releasedetail.cfm?ReleaseID = 1003098

48 Biogen presents data from Phase $1 \mathrm{~b}$ study of investigational Alzheimer's disease treatment aducanumab at 2016 Clinical Trials on Alzheimer's Disease Meeting. http://media.biogen.com/press-release/corporate/biogenpresents-data-phase-1b-study-investigational-alzheimersdisease-treat

49 Akcea and Ionis announce positive results from COMPASS Phase III study of volanesorsen. http://ir.ionispharma.com/phoenix. zhtml?c=222170 \&p=irol-newsArticle \&ID =2230503

50 ViiV Healthcare announces start of Phase III study evaluating long-acting cabotegravir for HIV prevention. www.viivhealthcare.com/media/press-releases/2016/ december/viiv-healthcare-announces-start-of-phase-iii-studyevaluating-long-acting-cabotegravir-for-hiv-prevention.aspx

51 ViiV Healthcare announces positive results from first Phase III studies of two-drug HIV treatment regimen. www.viivhealthcare.com/media/press-releases/2016/ december/viiv-healthcare-announces-positive-results-fromfirst-phase-iii-studies-of-two-drug-hiv-treatment-regimen. aspx

52 Opdivo (nivolumab) alone or combined with Yervoy (ipilimumab) shows encouraging response and survival rates in recurrent small cell lung cancer, From Phase 1/2 study CheckMate -032 .

http://news.bms.com/press-release/bmy/opdivo-nivolumabalone-or-combined-yervoy-ipilimumab-shows-encouragingresponse-an

53 Encouraging survival observed with Opdivo (nivolumab) plus Yervoy (ipilimumab) with longer follow-up in first-line advanced non-small cell lung cancer, in updated phase $1 \mathrm{~b}$ CheckMate -012 Study. http://news.bms.com/press-release/bmy/encouragingsurvival-observed-opdivo-nivolumab-plus-yervoyipilimumab-longer-follo 
54 Acadia Pharmaceuticals announces positive top-line results from Phase II study of pimavanserin for Alzheimer's disease psychosis.

http://ir.acadia-pharm.com/phoenix. zhtml? $c=125180 \& p=$ irol-newsArticle \&ID = 2230818

55 Acadia Pharmaceuticals initiates Phase II trial of pimavanserin for adjunctive treatment in patients with major depressive disorder.

http://ir.acadia-pharm.com/phoenix.

zhtml?c=125180\&p=irol-newsArticle \&ID =2226878

56 Loxo Oncology TRK inhibitor larotrectinib (LOXO-101) shows durable anti-tumor activity across TRK fusion cancers in ESMO Asia Phase 1 update.

https://ir.loxooncology.com/press-releases/loxo-oncologytrk-inhibitor-larotrectinib-loxo-101-shows-durable-antitumor-activity-across-trk-fusion-cancers-in-esmo-asia-phase1-update

57 Loxo Oncology outlines plans for accelerated path to U.S.
FDA approval for larotrectinib (LOXO-101) and provides comprehensive pipeline update.

https://ir.loxooncology.com/press-releases/loxo-oncologyoutlines-plans-for-accelerated-path-to-u.s.-fda-approval-forlarotrectinib-loxo-101-and-provides-comprehensive-pipelineupdate

Takeda and Seattle Genetics Report Positive Phase

III ALCANZA Clinical Trial Data of ADCETRIS ${ }^{\circledast}$

(Brentuximab Vedotin) for CD30-Expressing Cutaneous

T-Cell Lymphoma.

www.takeda.com/news/2016/20161204_7639.html

59 Seattle Genetics and Bristol-Myers Squibb highlight first data from Phase 1/2 study evaluating Adcentris ${ }^{\circledR}$ (brentuximab vedotin) in combination with Opdivo (nivolumab) in relapsed or refractory Hodgkin lymphoma at ASH annual meeting.

http://news.bms.com/press-release/bmy/seattle-genetics-andbristol-myers-squibb-highlight-first-data-phase-12-study-eval 
